\title{
Nonalcoholic Fatty Liver Disease, Cardiovascular Risk, and Carotid Inflammation
}

\author{
Khalid Al-Rasadi, MD', Manfredi Rizzo, MD, PhD', \\ Giuseppe Montalto, $\mathrm{MD}^{2}$, and Gabriela Berg, $\mathrm{PhD}^{3}$
}

Nonalcoholic fatty liver disease (NAFLD) is defined by excessive triglycerides (TGs) accumulation in the liver ( $>5 \%$ of hepatocytes histologically) in the absence of alcohol excess. ${ }^{1}$ The NAFLD ranges from simple steatosis to steatohepatitis and cirrhosis. ${ }^{1}$ The NAFLD and nonalcoholic steatohepatitis (NASH) are now the number one cause of liver disease in Western countries. ${ }^{2}$ The prevalence of NAFLD is increasing but is underreported, and the epidemiology and demographic characteristics vary worldwide. ${ }^{2}$ The prevalence is increasing because of the rising occurrence of obesity and type 2 diabetes (T2DM) ${ }^{1}$; in fact, NAFLD is considered as the hepatic manifestation of metabolic syndrome (MetS). ${ }^{3}$ Nonalcoholic fatty liver disease is present in $10 \%$ to $24 \%$ of the general population in various countries, ${ }^{2}$ while the prevalence of NAFLD in obesity is $30 \%$ to $100 \%$ and in T2DM is $10 \%$ to $75 \%{ }^{4}$ The morbidity and mortality from liver causes are increased in patients with NAFLD/NASH but is even stronger for cardiovascular disease (CVD). ${ }^{5}$ The association of NAFLD with carotid atherosclerosis ${ }^{6}$ and the increased risk of CVD in those with NAFLD and T2DM ${ }^{7}$ shed light on the association between NAFLD and CVD.

Atherosclerosis risk factors, such as hypertension, dyslipidemia, obesity, and insulin resistance (IR), frequently accompany NAFLD. ${ }^{7}$ However, the exact mechanisms involved remain unclear. Nowadays, 2 main factors are considered to contribute to the enhanced risk of CVD in persons with NAFLD, the lipoproteins synthesized by the liver and the increased visceral and ectopic adipose tissue promoting an inflammatory state. In obesity or IR conditions, excessive visceral adiposity increases the availability of free fatty acids (FFAs); this leads to enhanced hepatic production of very low-density lipoprotein (VLDL) and circulating TG levels. ${ }^{8}$

When evaluating patients with MetS, with and without steatohepatitis, those with NAFLD had increased circulating TG levels, higher VLDL mass, and VLDL number of particles. ${ }^{9,10}$ Atypical VLDL particles, enriched with TG, are also more atherogenic ${ }^{8}$; these particles have inhibitory effects on endothelial relaxation and are efficiently lipolyzed by lipoprotein lipase,${ }^{11}$ thus leading to the formation of smaller lipoproteins with atherogenic potential that can be further processed by hepatic lipase. ${ }^{12}$ Patients with NAFLD also had higher small dense low-density lipoprotein (sdLDL) concentration, associated with increased cholesterol ester transfer protein (CETP) concentration and hepatic lipase activity ${ }^{13}$ beyond IR; these factors contribute to a more atherogenic profile linked to increased CVD risk. Although the association between IR and increased LDL-cholesterol (LDL-C) levels is not typical, elevated sdLDL levels with lower large LDL concentrations are related to IR and increased adiposity. ${ }^{14}$ In contrast to large buoyant LDL, sdLDL particles are taken up more easily by arterial tissue and have greater oxidative and glycation susceptibility, suggesting a link with atherogenesis. ${ }^{15,16}$ Patients with high levels of sdLDL particles have an approximately 3- to 7-fold increased risk of developing coronary heart disease, independent of LDL-C concentration. ${ }^{17}$

Growing evidence suggests that the pathogenesis of NAFLD involves oxidative stress and inflammation. ${ }^{18}$ Patients with NAFLD present increased circulating concentrations of C-reactive protein (CRP) and FFA as well as reduced levels of adiponectin, independent of IR. ${ }^{19}$ In addition, high-sensitivity CRP (hsCRP) levels correlate with other inflammatory markers such as tumor necrosis factor $\alpha$ and with soluble cellular adhesion molecules such as vascular cell adhesion molecule 1 and intercellular adhesion molecule $1 .{ }^{19}$

Inflammation plays a key role in the initiation, progression, and rupture of atherosclerotic plaques. ${ }^{20}$ Furthermore, the inflammatory component of vulnerable plaques triggers events suggesting the need for research to understand the combined

\footnotetext{
'Department of Clinical Biochemistry, Sultan Qaboos University Hospital, Muscat, Oman

${ }^{2}$ Biomedical Department of Internal Medicine and Medical Specialties, University of Palermo, Palermo, Italy

${ }^{3}$ Department of Clinical Biochemistry, Faculty of Pharmacy and Biochemistry, University of Buenos Aires, Buenos Aires, Argentina
}

\section{Corresponding Author:}

Gabriela Berg, Department of Clinical Biochemistry, Faculty of Pharmacy and Biochemistry, Laboratory of Lipids and Atherosclerosis, Institute of Physiopathology and Clinical Biochemistry (INFIBIOC), Junin 956, Ciudad de Buenos Aires, Argentina.

Email: gaberg@ffyb.uba.ar 
role of inflammation and IR in the progression from subclinical to clinical atherosclerosis. ${ }^{21}$

Recent studies have shown that patients with NAFLD have significantly greater cIMT than age- and sex-matched patients without NAFLD, independent of the classical risk factors. ${ }^{22}$ Moreover, cIMT, assessed by ultrasound examination, is associated with the severity of liver histopathology among patients with NAFLD. ${ }^{22}$ It is of interest that statins may exert beneficial effects on cIMT and NAFLD. ${ }^{23-25}$

Nontraditional risk factors, as surrogate markers of cIMT, should also be considered, such as the recently proposed hsCRP-adiponectin ratio, a potential predictor of atherosclerosis progression. ${ }^{26}$ In controls and patients with MetS, the hsCRPadiponectin ratio was associated with circulating levels of matrix metalloproteinases (MMPs), enzymes that are associated with vulnerable plaques and IR conditions. ${ }^{27,28}$ Elevated serum MMP concentrations have been reported to be independently associated with carotid artery plaque instability and high cIMT value. ${ }^{29}$ Matrix metalloproteinases are activated in inflammatory states and $\mathrm{IR}^{28}$ and have also been proposed as surrogate markers of the severity of carotid artery disease. More recently, positron emission tomography with ${ }^{18} \mathrm{~F}$-fluorodeoxyglucose has been considered as a marker for active inflammation in atherosclerotic plaques in patients with NAFLD. ${ }^{30}$ It is important to decide whether further recommendations with regard to carotid atherosclerosis screening should be implemented in patients with NAFLD.

\section{Declaration of Conflicting Interests}

The author(s) declared no potential conflicts of interest with respect to the research, authorship, and/or publication of this article.

\section{Funding}

The author(s) received no financial support for the research, authorship, and/or publication of this article.

\section{References}

1. Farrell GC, Larter CZ. Nonalcoholic fatty liver disease: from steatosis to cirrhosis. Hepatology. 2006;43(2 suppl 1):s99-s112.

2. Angulo P. GI epidemiology: nonalcoholic fatty liver disease. Aliment Pharmacol Ther. 2007;25(8):883-889.

3. Musso G, Gambino R, Bo S, et al. Should nonalcoholic fatty liver disease be included in the definition of metabolic syndrome? A cross-sectional comparison with adult treatment panel III criteria in nonobese nondiabetic. Diabetes Care. 2008;31(3):562-568.

4. Ahmed MH, Barakat S, Almobarak AO. Nonalcoholic fatty liver disease and cardiovascular disease: has the time come for cardiologists to be hepatologists? J Obes. 2012;2012:483135. doi:10. 1155/2012/483135.

5. Monsour HP Jr, Frenette CT, Wyne K. Fatty liver: a link to cardiovascular disease - its natural history, pathogenesis, and treatment. Methodist Debakey Cardiovasc J. 2012;8(3):21-25.

6. Brea A, Mosquera D, Martín E, Arizti A, Cordero JL, Ros E. Nonalcoholic fatty liver disease is associated with carotid atherosclerosis: a case-control study. Arterioscler Thromb Vasc Biol. 2005;25(5):1045-1050.
7. Targher G, Bertolini L, Poli F, et al. Nonalcoholic fatty liver disease and risk of future cardiovascular events among type 2 diabetic patients. Diabetes. 2005;54(12):3541-3546.

8. Nikolic D, Katsiki N, Montalto G, Isenovic ER, Mikhailidis DP, Rizzo M. Lipoprotein subfractions in metabolic syndrome and obesity: clinical significance and therapeutic approaches. Nutrients. 2013;5(3):928-948.

9. Lucero D, Zago V, López GI, et al. Does non-alcoholic fatty liver impair alterations of plasma lipoproteins and associated factors in metabolic syndrome? Clin Chim Acta. 2011;412(7-8):587-592.

10. Lucero D, Zago V, López GH, et al. Predominance of large VLDL particles in metabolic syndrome, detected by size exclusion liquid chromatography. Clin Biochem. 2012;45(4-5):293-297.

11. Zago V, Gorzalczany S, Lucero D, Taira C, Schreier L. Role of HDL in neutralizing the VLDL effect on endothelial dysfunction. Microvasc Res. 2013;89:153-158.

12. Rizzo M, Taylor JM, Barbagallo CM, Berneis KK, Blanche PJ, Krauss RM. Effects on lipoprotein subclasses of combined expression of human hepatic lipase and human apoB in transgenic rabbits. Arterioscler Thromb Vasc Biol. 2004;24(1):141-146.

13. Miksztowicz V, Lucero D, Zago V, et al. Hepatic lipase activity is increased in non-alcoholic fatty liver disease beyond insulin resistance. Diabetes Metab Res Rev. 2012;28(6):535-541.

14. Rizzo M, Rini GB, Berneis K. The clinical relevance of LDL size and subclasses modulation in patients with type-2 diabetes. Exp Clin Endocrinol Diabetes. 2007;115(8):477-482.

15. Rizzo M, Mikhailidis DP. There is more to predicting vascular disease than just established risk factors. Curr Pharm Des. 2011;17(33):3608-3610.

16. Rizzo M, Pernice V, Frasheri A, Berneis K. Atherogenic lipoprotein phenotype and LDL size and subclasses in patients with peripheral arterial disease. Atherosclerosis. 2008;197(1):237-241.

17. Mikhailidis DP, Elisaf MS, Rizzo M, et al. "European Panel on Low Density Lipoprotein (LDL) Subclasses": a statement on the pathophysiology, atherogenicity and clinical significance of LDL subclasses. Curr Vasc Pharmacol. 2011;9(5):533-571.

18. Cortez-Pinto H, de Moura MC, Day CP. Non-alcoholic steatohepatitis: from cell biology to clinical practice. J Hepatol. 2006; 44(1):197-208.

19. Lucero D, Zago V, López GI, et al. Pro-inflammatory and atherogenic circulating factors in non-alcoholic fatty liver disease associated to metabolic syndrome. Clin Chim Acta. 2011;412(1-2): 143-147.

20. Ross R. Atherosclerosis is an inflammatory disease. Am Heart J. 1999;138(5 Pt 2):S419-S420.

21. Corrado E, Rizzo M, Coppola G, Muratori I, Carella M, Novo S. Endothelial dysfunction and carotid lesions are strong predictors of clinical events in patients with early stages of atherosclerosis: a 24-month follow-up study. Coron Artery Dis. 2008;19(3):139-144.

22. Sookoian S, Pirola CJ. Non-alcoholic fatty liver disease is strongly associated with carotid atherosclerosis: a systematic review. J Hepatol. 2008;49(4):600-607.

23. Peters SA, den Ruijter HM, Bots ML. Attenuation of rate of change in carotid intima-media thickness by lipid-modifying drugs: impact on clinical outcomes. Am J Cardiovasc Drugs. 2011;11(4):253-263. 
24. Athyros VG, Tziomalos K, Gossios TD, et al; GREACE Study Collaborative Group. Safety and efficacy of long-term statin treatment for cardiovascular events in patients with coronary heart disease and abnormal liver tests in the Greek Atorvastatin and Coronary Heart Disease Evaluation (GREACE) Study: a posthoc analysis. Lancet. 2010;376(9756):1916-1922.

25. Tikkanen MJ, Fayyad R, Faergeman O, et al; IDEAL Investigators. Effect of intensive lipid lowering with atorvastatin on cardiovascular outcomes in coronary heart disease patients with mild-to-moderate baseline elevations in alanine aminotransferase levels. Int J Cardiol. 2013;168(4):3846-3852.

26. Liao H, Li Z, Zheng D, et al. Increased Hs-CRP/adiponectin ratio is associated with increase carotid intima-media thickness. Lipids Health Dis. 2014;13:120.
27. Miksztowicz V, Fernandez Machulsky N, et al. Adiponectin predicts MMP-2 activity independently of obesity. Eur J Clin Invest. 2014;44(10):951-957.

28. Berg G, Miksztowicz V, Schreier L. Metalloproteinases in metabolic syndrome. Clin Chim Acta. 2011;412(19-20):1731-1739.

29. Tan C, Liu Y, Li W, et al. Associations of matrix metalloproteinase-9 and monocyte chemoattractant protein-1 concentrations with carotid atherosclerosis, based on measurements of plaque and intima-media thickness. Atherosclerosis. 2014;232(1):199-203.

30. Moon SH, Noh TS, Cho YS, et al. Association between nonalcoholic fatty liver disease and carotid artery inflammation evaluated by $18 \mathrm{~F}$-fluorodeoxyglucose positron emission tomography. Angiology. 2015;66(5):472-480. 